\title{
Two Alternative Proofs of the Grüss Inequality
}

\author{
Martin Tchernookov
}

Physics Department, University of Wisconsin Whitewater

\footnotetext{
* Corresponding author email: martin.whitewater@gmail.com

Received: 09 September 2020 / Revised: 07 October 2020 / Accepted: 13 October 2020 / Published: 21 October 2020
}

\begin{abstract}
The classical Grüss inequality has spurred a range of improvements, generalizations, and extensions. In this article, we provide new functional bounds that ultimately lead to two elementary proofs of the inequality that might be of interest. Our results are motivated by the extreme cases where the equality is reached, namely step functions of equal support. Our first proof is based on the standard Cauchy-Schwarz inequality and a simple bound on the variance of a function. Its simplicity would be of particular interest to those who are new to the study of functional inequalities. Our second proof utilizes non-intuitive and novel bounds on functionals defined on $L^{\infty}(0,1)$. As a result, we provide a detailed and new insight into the nature of the Grüss inequality.

Keywords: Inequalities, Gruss inequality, Functional inequalities.
\end{abstract}

\section{Introduction}

Grüss demonstrated an interesting and rather useful functional inequality defined on essentially bounded, measurable functions that controls the difference between the integrated product of two such functions and the product of their integrals. It has, since, generated a significant amount of additional results. The Grüss inequality has some simple extensions [1]. More complex generalizations are also well-known [2, 3] (see [4] for an interesting proof of the Ostrowski-Grüss inequality). These generalizations have found significant applications in numerical mathematical analysis, probability and statistics, and coding theory.

The classical Grüss inequality [5] deals with $L^{\infty}(a, b)$, the space of real-valued, Lebesgue measurable, essentially bounded functions defined on the interval $[a, b] \subset \mathbb{R}$ :

Copyright (C) 2020. The Author(s). Published by AIJR Publisher.

This is an open access article under Creative Commons Attribution- NonCommercial 4.0 International (CC BY-NC 4.0) license, which permits any non-commercial use, distribution, adaptation, and reproduction in any medium, as long as the original work is properly cited. 
Theorem 1. Let $f, g \in L^{\infty}(a, b)$ be such that $m \leq f \leq M$ and $p \leq g \leq P$ almost everywhere. Then,

$$
\begin{aligned}
& \frac{1}{b-a} \int_{a}^{b} f(x) g(x) d \lambda(x)-\frac{1}{(b-a)^{2}} \int_{a}^{b} f(x) d \lambda(x) \int_{a}^{b} g(x) d \lambda(x) \\
& \leq \frac{(M-m)(P-p)}{4} .
\end{aligned}
$$

Proofs of (1) are not elementary [6, 7]. In the following paper we present two additional, self-contained proofs that might be of interest. In particular, we provide additional insight and interpretation into why the inequality holds.

It is straightforward to check that (1) can be re-written as

$$
\frac{1}{(b-a)^{2}} \int_{a}^{b} d \lambda(x) \int_{a}^{b} d \lambda(y)\left[\frac{f(x)-m}{M-m}-\frac{f(y)-m}{M-m}\right]\left[\frac{g(x)-p}{P-p}-\frac{g(y)-p}{P-p}\right] \leq \frac{1}{2} .
$$

Notice that the transformation $(f(x)-m) /(M-m)$ is a bijection from $\left\{f \in L^{\infty}(a, b) \mid m \leq\right.$ $f \leq M$, a.e. $\}$ to $\left\{f \in L^{\infty}(a, b) \mid 0 \leq f \leq 1\right.$, a.e. $\}$. A similar statement is true for the transformation $(g(x)-p) /(P-p)$. Therefore, the left hand side of (2) involves functions with essential bounds 0 and 1 , while the right hand side is a constant. These observations, coupled with the transformation $x \rightarrow(x-a) /(b-a)$, define the shift and scale invariance of (1). Therefore, it is actually convenient to consider, without loss of generality, the specialization $[a, b]=[0,1]$ and $[m, M]=[p, P]=[0,1]$. In this case, therefore, we can re-write (1) as

$$
\int_{0}^{1} d \lambda(x) \int_{0}^{1} d \lambda(y)(f(x)-f(y))(g(x)-g(y)) \leq \frac{1}{2} .
$$

The rest of the paper centers on proving (3). The main results of the paper are shown below. We will use these results to prove theorem 1 and then we will prove the results themselves. In the following, sets are measurable in the Lebesgue sense and $\lambda$ refers to the Lebesgue measure on the real line.

Lemma 1. Let $X \subset \mathbb{R}$ be measurable with $\lambda(X)=1$ and let $f: X \rightarrow[m, M]$ with $\int_{X} f(x) d \lambda(x)=\mu$. Then,

$$
\int_{X}(f(x)-\mu)^{2} d \lambda(x) \leq(M-\mu)(\mu-m) .
$$

Lemma 2. Let $X \subset[0,1]$ be measurable and $f: X \rightarrow \mathbb{R}$ integrable. Then, we can find a subset $S \subset X$ with $\lambda(S)=\lambda(X) / 2$, together with a real number $\kappa$ such that $f \leq \kappa$ on $S$ and $f \geq \kappa$ on $S^{c}$. 
Theorem 2. Let $X \subset[0,1]$ with $\lambda(X)=1$ and let $f: X \rightarrow[-1,1]$ be a measurable function. Then,

$$
\int_{X} \int_{X}|f(x)-f(y)| d \lambda(x) d \lambda(y) \leq 1
$$

\section{First proof of the Grüss inequality}

The main motivation behind this proof is the fact that the left hand side of (1) resembles the correlation function between two random variables. It involves the average of the product of $f$ and $g$, from which the product of the individual averages is subtracted. Well-known bounds on the correlation function can then be invoked. Using the CauchySchwarz inequality, we can bound the left hand side of (3) as follows

$$
\int_{0}^{1} d \lambda(x) \int_{0}^{1} d \lambda(y)(f(x)-f(y))(g(x)-g(y)) \leq \sqrt{T(f) T(g)}
$$

where the functional $T$ is defined as

$$
T(f)=\int_{0}^{1} d \lambda(x) \int_{0}^{1} d \lambda(y)(f(x)-f(y))^{2} .
$$

We can further expand the right hand side of the above definition and apply lemma 1

$$
\begin{aligned}
& \int_{0}^{1} d \lambda(x) \int_{0}^{1} d \lambda(y)(f(x)-f(y))^{2}=2 \int_{0}^{1} f(x)^{2} d \lambda(x)-2\left(\int_{0}^{1} f(x) d \lambda(x)\right)^{2} \\
& =2 \int_{0}^{1}(f(x)-\mu)^{2} d \lambda(x) \leq 2 \mu(1-\mu) \leq \frac{1}{2}
\end{aligned}
$$

where $\mu$ is as defined in lemma 1 . It is clear now that (7) and (5) imply (3).

\section{Second proof of the Grüss inequality}

A more direct way to prove (1) is presented in this section. A naive bound on the left hand side of (3), that replaces the $g(x)-g(y)$ factor under the integral with its maximum possible value, leads to

$$
\int_{0}^{1} d \lambda(x) \int_{0}^{1} d \lambda(y)(f(x)-f(y))(g(x)-g(y)) \leq \int_{0}^{1} \int_{0}^{1}|f(x)-f(y)| d \lambda(x) d \lambda(y) .
$$


Martin Tchernookov, Int. Ann. Sci.; Vol. 10, Issue 1, pp: 78-84, 2021

The inequality (3) follows now directly from (8) and theorem 2. The bulk of this proof is in lemma 2 and theorem 2. Notice that dropping $g(x)-g(y)$ in the integrand of (3) did not lead to a weaker inequality; indeed, it allowed us to prove it. This is not accidental; indeed, the reason, and also the motivation for this proof, is in the extreme cases which reduce (1) to an identity, namely, when $f$ and $g$ are equal to the same step function. As an example, consider the case

$$
f(x)=g(x)= \begin{cases}0, & x<1 / 2 \\ 1, & x \geq 1 / 2\end{cases}
$$

It is straightforward to check that these functions reach the equality in (3). More importantly, equality is also reached in (8), lemma 1 , and theorem 2 . The choice above is not unique; any step function with equal support, i.e. $\lambda\left(f^{-1}(0)\right)=\lambda\left(f^{-1}(1)\right)$, will reach the equality in (3). How this happens is demonstrated in the proof of theorem 2.

\section{Proofs of the main results}

Proof of lemma 1. Let $S=\{x \in X \mid f(x) \geq \mu\}$, a measurable subset of $X$. We have

$$
\int_{S}(f(x)-\mu)^{2} d \lambda(x) \leq(M-\mu) \int_{S}(f(x)-\mu) d \lambda(x) .
$$

Similarly on the complement of $S$, we have

$$
\int_{S^{c}}(f(x)-\mu)^{2} d \lambda(x) \leq(m-\mu) \int_{S^{c}}(f(x)-\mu) d \lambda(x) .
$$

Adding (9) and (10) and taking into consideration the definition of $\mu$, we obtain

$$
\int_{X}(f(x)-\mu)^{2} d \lambda(x) \leq(M-m) \int_{S}(f(x)-\mu) d \lambda(x) .
$$

On the other hand, the boundedness of $f(x)-\mu$ on $S$ and $S^{c}$ implies the following sequence of inequalities

$$
\begin{aligned}
& \lambda\left(S^{c}\right) \geq \frac{1}{\mu-m} \int_{S^{c}}(\mu-f(x)) d \lambda(x) ; \\
& \int_{S}(f(x)-\mu) d \lambda(x) \leq(M-\mu) \lambda(S)=(M-\mu)\left(1-\lambda\left(S^{c}\right)\right) \\
& \leq(M-\mu)\left(1-\frac{1}{\mu-m} \int_{S}(f(x)-\mu) d \lambda(x)\right) \\
& \Rightarrow \int_{S}(f(x)-\mu) d \lambda(x) \leq \frac{(M-\mu)(\mu-m)}{M-m} .
\end{aligned}
$$

Plugging inequality (12) into the right side of (11) proves the lemma. 
Proof of lemma 2. We start the proof with the observation that for any real number $k$, we have the simple set identities $f^{-1}((-\infty, k))=\bigcup_{l<k} f^{-1}((-\infty, l))$ and $f^{-1}((-\infty, k])=$ $\bigcap_{l>k} f^{-1}((-\infty, l))$. We then define the function $\phi(k) \equiv \lambda\left(f^{-1}((-\infty, k))\right)$. From the above set identities and the monotonicity of the Lebesgue measure, it follows that $\phi$ is a monotonically increasing, left continuous function with a potential jump at a given real number $k$ equal to $\lambda\left(f^{-1}(k)\right)$. From the integrability of $f$, it follows that $\lim _{k \rightarrow-\infty} \phi(k)=0$ and $\lim _{k \rightarrow \infty} \phi(k)=\lambda(X)$. If there is a real number $\kappa$ such that $\phi(\kappa)=\lambda(X) / 2$, we can define $S \equiv f^{-1}((-\infty, \kappa))$ and the proof of the lemma would be complete.

Otherwise, assume that such a real number does not exist and define $\kappa=\sup \{k \mid \phi(k) \leq$ $\lambda(X) / 2\}$. From the left continuity and this assumption, we have $\phi(\kappa)<\lambda(X) / 2$. From the definition of $\kappa$ it also follows that $\lim _{k \rightarrow \kappa+} \phi(k) \geq \lambda(X) / 2$. Thus, we can find a number $r \in(0,1]$ such that the set

$$
S \equiv f^{-1}((-\infty, \kappa)) \bigcup\left\{f^{-1}(\kappa) \bigcap[0, r]\right\}
$$

satisfies $\lambda(S)=\lambda(X) / 2$. It is obvious that $f\left(S^{c}\right) \subset[\kappa, \infty)$ and the proof of the lemma is now finished.

Proof of theorem 2. Let $S, S^{c}$, and $\kappa$ be as in lemma 2. We can divide the integral in the statement of theorem 2 as follows

$$
\begin{aligned}
& \int_{X} \int_{X}|f(x)-f(y)| d \lambda(x) d \lambda(y)=\int_{S} \int_{S}|f(x)-f(y)| d \lambda(x) d \lambda(y) \\
& +\int_{S^{c}} \int_{S^{c}}|f(x)-f(y)| d \lambda(x) d \lambda(y)+2 \int_{S} \int_{S^{c}}|f(x)-f(y)| d \lambda(x) d \lambda(y) .
\end{aligned}
$$

We will use this breakdown to construct a modified function that reaches equality in (4). We start by modifying $f$ over the set $S^{c}$. To this end, define the function

$$
\tilde{f}(x)= \begin{cases}f(x), & x \in S \\ 1, & x \in S^{c}\end{cases}
$$

The function $\tilde{f}$ is constant on $S^{c}$, hence

$$
\int_{S^{c}} \int_{S^{c}}|\tilde{f}(x)-\tilde{f}(y)| d \lambda(x) d \lambda(y)=0
$$


More importantly, $\tilde{f}(y) \geq \kappa \geq \tilde{f}(x)$ for any $x \in S$ and $y \in S^{c}$. Therefore, we have

$$
\begin{aligned}
& \int_{S} \int_{S^{c}}|\tilde{f}(x)-\tilde{f}(y)| d \lambda(x) d \lambda(y)=\int_{S} d \lambda(x) \int_{S^{c}} d \lambda(y)(1-f(x))= \\
& \int_{S} d \lambda(x) \int_{S^{c}} d \lambda(y)[1-f(y)+f(y)-f(x)]= \\
& \frac{1}{2}\left(\frac{1}{2}-\int_{S^{c}} f(x) d \lambda(x)\right)+\int_{S} d \lambda(x) \int_{S^{c}} d \lambda(y)|f(x)-f(y)| .
\end{aligned}
$$

Lastly, we can write

$$
\begin{aligned}
& \int_{S^{c}} \int_{S^{c}}|f(x)-f(y)| d \lambda(x) d \lambda(y)=\int_{S^{c}} \int_{S^{c}}|(1-f(y))-(1-f(x))| d \lambda(x) d \lambda(y) \leq \\
& 2 \int_{S^{c}} \int_{S^{c}}|1-f(x)| d \lambda(x) d \lambda(y)=\int_{S^{c}}(1-f(x)) d \lambda(x)=\frac{1}{2}-\int_{S^{c}} f(x) d \lambda(x) .
\end{aligned}
$$

Combining (14), (15), and (16) into the breakdown (13) proves that

$$
\int_{X} \int_{X}|\tilde{f}(x)-\tilde{f}(y)| d \lambda(x) d \lambda(y) \geq \int_{X} \int_{X}|f(x)-f(y)| d \lambda(x) d \lambda(y) .
$$

We can re-apply the above logic to the functions $\tilde{f}(x)$ and

$$
\phi(x)= \begin{cases}-1, & x \in S \\ 1, & x \in S^{c}\end{cases}
$$

to obtain that

$$
\int_{X} \int_{X}|\tilde{f}(x)-\tilde{f}(y)| d \lambda(x) d \lambda(y) \leq \int_{X} \int_{X}|\phi(x)-\phi(y)| d \lambda(x) d \lambda(y)=1 .
$$

Inequalities (17) and (18) imply the theorem.

\section{Conclusion}

In this paper, we provided unique and self-contained results that provide bounds on functions defined on the real line with very few conditions. These results lead to two proofs of the classical Grüss inequality. Our first proof was based on the standard CauchySchwartz inequality and a simple bound on the variance of a function. We believe that this would be of particular interest to those who are new to the study of functional inequalities. Our second proof utilized non-intuitive and novel bounds on functionals defined on $L^{\infty}(0,1)$. Through the use of equally supported step functions, in conjunction with the above bounds, we provided a detailed and new insight into the nature of the Grüss inequality. 
Two Alternative Proofs of the Grüss Inequality

\section{Competing Interests}

The author declares that there are no conflicts of interest regarding the publication of this paper.

\section{How to Cite this Article:}

M. Tchernookov, "Two Alternative Proofs of the Grüss Inequality", Int. Ann. Sci., vol. 10, no. 1, pp. 78-84, Oct. 2020. https://doi.org/10.21467/ias.10.1.78-84

\section{References}

[1] Mercer, A M.: An improvement of the Grüss inequality. In: JIPAM. J. Inequal. Pure Appl. Math 6 (2005), Nr. 4, S. 93

[2] Dragomir, Sever S.: Some Grüss-type results via Pompeiu's-like inequalities. In: Arabian Journal of Mathematics 4 (2015), Nr. 3, 159-170. http://dx.doi.org/10. 1007/s40065-015-0135-8. - DOI 10.1007/s40065-015-0135-8

[3] Niezgoda, Marek: A new inequality of Ostrowski-Grüss type and applications to some numerical quadrature rules. In: Computers 83 Mathematics with Applications 58 (2009), Nr. 3, 589-596. http://dx.doi.org/10.1016/j.camwa.2009.03.089. DOI 10.1016/j.camwa.2009.03.089

[4] FARID, Ghulam u. a.: Straightforward proofs of Ostrowski inequality and some related results. In: Int. J. Ana 5 (2016). http://dx.doi.org/10.1155/2016/3918483. DOI $10.1155 / 2016 / 3918483$

[5] GRÜss, Gerhard: Über das Maximum des absoluten Betrages von. In: Mathematische Zeitschrift 39 (1935), Nr. 1, 215-226. http://dx.doi.org/10.1007/BF01201355. DOI $10.1007 /$ BF 01201355

[6] Mercer, A M. ; Mercer, PR: New proofs of the Grüss inequality. In: Aust. J. Math. Anal. Appl 1 (2004), Nr. 2, S. 6

[7] Mitrinovic, Dragoslav S. ; Pecaric, Josip ; Fink, Arlington M.: Classical and new inequalities in analysis. Bd. 61. Springer Science \& Business Media, 2013. http://dx.doi.org/10.1007/978-94-017-1043-5. http://dx.doi.org/10.1007/ 978-94-017-1043-5 